\title{
A Microscopic Approach for Generic Degradation Modeling
}

\author{
Farhang Momeni ${ }^{1}$, Xiaoning $\mathrm{Jin}^{2}$, and $\mathrm{Jun}_{\mathrm{Ni}}{ }^{1}$ \\ ${ }^{1}$ Department of Mechanical Engineering, University of Michigan, Ann Arbor, Michigan 48109, USA \\ farhang@umich.edu \\ junni@umich.edu \\ ${ }^{2}$ Department of Mechanical and Industrial Engineering, Northeastern University, Boston, Massachusetts, 01890, USA \\ xi.jin@northeastern.edu
}

\begin{abstract}
One of the main challenges in prognostics is degradation modeling for which there are no straightforward methods compared with system governing equations modeling (e.g., Newton`s law, Euler's law, thermodynamics conservation laws, fluid dynamics laws, and so forth). Once both governing equations and degradation equations are generated, then the RUL or EOL can be easily estimated by using a filtering technique like Kalman filter. This paper presents a new approach for generic degradation modeling which can be engaged in (1) complex engineering systems, and (2) the structures which are fabricated by the new manufacturing processes such as $4 \mathrm{D}$ printing that in both cases the physical knowledge is not adequate to model the degradation equations. In the existing approaches for parametric degradation modeling, there are always possibilities that a specific degradation phenomenon of a new system is ignored. This deficiency arises from the assumption made in the previous studies that degradation phenomenon is equivalently represented by degradation mechanism such as crack, wear, corrosion, erosion, and so forth. Here we relax this assumption and provide a more general approach for parametric degradation modeling. This research first, quantifies the concepts of governing equations and degradation equations in prognostics in a general view, second provides a generic approach for deriving the governing equations by using techniques of system identification, third, it digs into the concepts of degradation and provides a microscopic approach for generic degradation modeling, and finally it combines and incorporates the governing equations and degradation equations in a way that the generality is conserved. In addition, this paper opens the door of degradation modeling in $4 \mathrm{D}$ printing by starting from the conceptual point of view to the final generic methodology.
\end{abstract}

Farhang Momeni et al. This is an open-access article distributed under the terms of the Creative Commons Attribution 3.0 United States License, which permits unrestricted use, distribution, and reproduction in any medium, provided the original author and source are credited.

\section{INTRODUCTION}

Degradation modeling is one of the most challenging parts of prognostics. Degradation modeling is different from system dynamic (governing equations) modeling. For the latter, there are always some certain physical rules like Newton's law, Euler's law, Thermodynamics conservation laws, and so forth. However, for degradation modeling, there are no straightforward methods (like balancing the moments and forces which are done in deriving the governing equations) to represent a degradation model that governs the degradation behavior of a system.

There are mainly two categories of approach in the literature of prognostics - Physics-based and Data-driven approaches. The latest comprehensive review works in prognostics that have been done by Liao and Kottig (2014) and Aizpurua and Catterson (2015), introduced all types of techniques in physics-based and data-driven approaches up to their date perfectly. One big issue in the most of the previous works in parametric degradation modeling is that always there is the possibility that one specific degradation phenomenon is ignored. This issue arises when degradation phenomena are equated with some well-known degradation mechanisms like crack, wear, corrosion, erosion, and so forth. In this paper, we dig into the concepts of degradation and provide a microscopic approach in degradation modeling to provide a more complete set of degradation equations that consequently leads to a more reliable RUL estimation. Finally, to illustrate the capability of this approach in practice, two cases including one case in traditional degradation modeling and one case in $4 \mathrm{D}$ printing are discussed in the section of discussion. 4D printing is a new and fascinating field, and the 4D printed structure has targeted shape-shifting through time in contrast to 3D printed structures (Tibbits, 2014). Degradation is one of the main challenges in $4 \mathrm{D}$ printing so that after a certain number of shape-shifting, the structure would not entirely recover its original shape and thus, it is important to have an understanding of the degradation and the lifespan of the structure (Raviv, Zhao, McKnelly, Papadopoulou, Kadambi, 
Shi, Hirsch, Dikovsky, Zyracki, Olguin, Raskar, \& Tibbits 2014).

Although the necessity of degradation modeling in 4D printing has been addressed (Tibbits, McKnelly, Olguin, Dikovsky, \& Hirsch, 2014) and (Raviv et al. 2014), but no attempt has been made further to yield an appropriate degradation modeling for this new area.

The current parametric degradation modeling considering the well-known degradation mechanisms such as wear, corrosion, etc. are not suitable to model the degradation of the $4 \mathrm{D}$ printed structures. Instead, here, a more general approach based on a better concept, degradation phenomenon, is proposed to be beneficial for degradation modeling of $4 \mathrm{D}$ printed structures.

\section{QUANTIFYING THE CONCEPTS OF GOVERNING EQUATIONS AND DEGRADATION EQUATIONS IN PROGNOSTICS}

Here, we want to revisit the prognostics and degradation, more conceptually that creates the basis of our method. Assume that we have a system, and the system has $P$ system equations $e q_{1}, e q_{2}, \ldots, e q_{P}$. In these equations, we have $N$ input variables $U(t), M$ output variables $Y(t)$, and $K$ system parameter variables $\Theta(t)$. Parameters will change over time, but we may have some parameters that are fixed over time.

In general, each equation of the system, $e q_{1}, e q_{2}, \ldots, e q_{P}$ represents a relationship of inputs, outputs, and parameters. So we have:

$$
\left\{\begin{array}{c}
e q_{1}=e q_{1}(U(t), Y(t), \Theta(t)) \\
e q_{2}=e q_{2}(U(t), Y(t), \Theta(t)) \\
\cdot \\
\cdot \\
e q_{P}=e q_{P}(U(t), Y(t), \Theta(t))
\end{array}\right.
$$

where:

$$
\left\{\begin{array}{l}
\Theta(t)=\left[\theta_{1}(t), \theta_{2}(t), \ldots, \theta_{K}(t)\right]^{T} \\
U(t)=\left[u_{1}(t), u_{2}(t), \ldots, u_{N}(t)\right]^{T} \\
Y(t)=\left[y_{1}(t), y_{2}(t), \ldots, y_{M}(t)\right]^{T}
\end{array}\right.
$$

Finally, some new variables are defined and are called state variables. State variables are just some combinations (relationships) of the previous variables (inputs, outputs, and system parameters) and their trends are monitored until some prescribed thresholds are violated that it leads to RUL or EOL estimation. So assume we have $R$ desired state variables, $X(t)=\left[x_{1}(t), x_{2}(t), \ldots, x_{R}(t)\right]^{T}$.

Once the system governing equations (differential or algebraic) have been established to represent the system behavior, the parameters need to be identified. Some parameters are constant with time and some others change/evolve over time, related to certain degradation or gradual changes in the system. The assumption of constant parameters is useful in some cases when operation time is short (relative to lifetime) like for control purposes. However, for prognostics problems in which system could experience gradual and slow degradation, it is important to determine the degradation equations, which are some extra equations (secondary equations) that show the changes (drifts) in system parameters (not necessarily all parameters) over time. Hence assume that we have $Q$ degradation equations (degradation models) in a general form of Eq. (3) which consists of inputs, outputs, system parameters, and some new parameters that we call them secondary parameters that are showed up in the degradation (secondary) equations and not in primary system equations. So assume that we have $V$ secondary parameters, $h_{1}(t) \ldots h_{V}(t)$, the degradation equations can be written in the following form:

$$
\left\{\begin{array}{c}
e q_{P+1}=e q_{P+1}(U(t), Y(t), \Theta(t), \mathrm{H}(t)) \\
e q_{P+2}=e q_{P+2}(U(t), Y(t), \Theta(t), \mathrm{H}(t)) \\
\cdot \\
\cdot \\
e q_{P+Q}=e q_{P+Q}(U(t), Y(t), \Theta(t), \mathrm{H}(t)),
\end{array}\right.
$$

where:

$$
H(t)=\left[h_{1}(t), h_{2}(t), \ldots, h_{V}(t)\right]^{T} .
$$

By knowing all $P$ system equations and $Q$ degradation equations, we can proceed to RUL or EOL estimation. The examples of parametric degradation equations can be found in many prognostic studies, like those have been used by Daigle and Goebel (2011) and Daigle, Saha, and Goebel (2012). However, we will discuss the degradation equations in more details in section 4 to build our methodology and show the difference between our parametric degradation modeling and existing parametric degradation modeling studies.

\section{A GENERIC APPROACH FOR DERIVING THE GOVERNING EQUATIONS}

For many complex engineering systems, physical knowledge of the system is unknown or not enough to generate the governing equations, so physics-based approach cannot be helpful to generate all governing equations, but assuming generating all equations including the degradation models is in one's interest. In such situations, the data-driven approach cannot be helpful as well. In the works related to data-driven approaches, there is no comprehensive methodology to show a way of deriving all governing equations of the system for all applications. Here, a new approach is presented that is the basis of next section and will be incorporated into it later. Suppose that we have the Mass-Spring-Damper System Excited by Force $F(t)$, By balancing the forces, we can generate the 
differential equations and by taking a Laplace transformation, we have:

$$
G(s)=\frac{x}{F}=\frac{1}{m s^{2}+c s+k}
$$

where $F$ is the input, and $x$ is the output. The system parameters $m, c$, and $k$ are known from the physical knowledge of the system. By substituting the values of parameters, the transfer function becomes:

$$
G(s)=\frac{x}{F}=\frac{1}{5 s^{2}+20 s+1000}
$$

Now assume that we cannot find above equation by physics, but we have some data as inputs and outputs, in this condition, we can find the transfer function of $G(s)$ by system identification techniques. However, first, we need to find the order of the model. Every transfer function in the most general form is like below:

$$
G(s)=\frac{a_{0}+a_{1} s+a_{2} s^{2}+a_{3} s^{3}+\ldots+a_{n} s^{n}}{b_{0}+b_{1} s+b_{2} s^{2}+b_{3} s^{3}+\ldots+b_{m} s^{m}}
$$

So before we proceed to find the parameters, we need to find the model order, i.e., the values of $n$ and $m$ in Eq. (7).

\subsection{Model order estimation}

As reported by Ljung (1999), model order estimation mainly falls into the four categories: (1) Investigating the spectral analysis of the transfer function, (2) Investigating the ranks of sample covariance matrices, (3) Correlating variables, (4) Investigating the information matrix (Ljung, 1999). For further details, one can see the aforementioned reference.

\subsection{Model identification}

After identifying the model order, we need to find the parameters of the transfer function. We use model identification techniques that have been well developed in liteature, i.e., Ljung (2009) and Garnier, Mensler, and Richard (2003). Also, we should mention that these techniques are used to find constant parameters.

However, in the procedure of determining the system parameters, the model structure needs to be determined as described below.

\subsection{Model Structure Estimation}

Ljung (2013) explained that the linear models often could be adequate to show the system behavior, but if in some applications the model output does not appropriately regenerate the measured output, then we need to utilize a nonlinear model.

Determining that the model is linear or nonlinear is called Model Structure estimation, that naturally is before the
Model Order Estimation, but we usually start with linear structure and then we try to estimate the model order and if the behavior of the system could not be fitted to inputs and outputs appropriately, then we need to try some certain nonlinear models like Nonlinear ARX Models, Hammerstein-Wiener Models, and Linear Approximation of Nonlinear Models (Ljung, 2013) and (Ljung, 1999). So in the previous example, after estimating the model structure and model order, then we will reach to following transfer function:

$$
G(s)=\frac{x}{F}=\frac{b_{1}}{a_{1} s^{2}+a_{2} s+a_{3}}
$$

Moreover, as we discussed earlier, we can find the parameters $a_{1}, a_{2}, a_{3}, b_{1}$ by using model identification and finally we reach to same Eq. (6).

As we see, the final result in physics-based approach and this approach (up to here) is identical (a transfer function with known parameters).

To fulfill the modeling sub-part of the prognosis, we need to proceed to one more step that is model validation.

\subsection{Model Validation}

In the physics based approach, we usually do not have this step, because the governing equations have been generated with universally accepted principal laws, e.g., Newton's or Euler's laws have been accepted, but here we need model validation because the system equations have been generated by using measured data.

For validating model, there are some certain methods to validate both linear and nonlinear models, and one can see (Ljung, 2013).

So after we confirmed that our model is suitable, we can proceed to the next step. Otherwise, we need to return and regenerate proper system equations.

Finally, we have the system equations (primary equations), the second step is finding the degradation equations (secondary equations).

\section{A GENERIC APPROACH FOR DERIVING THE DEGRADATION EQUATIONS}

Degradation equations can be any function of inputs, outputs, and parameters. Parametric degradation modeling has some drawbacks. First, it is necessary to decide which one of inputs, outputs, and system parameters is effective in each degradation model. Second, it is necessary to decide the form of relationship between those chosen variables. Third, ensuring that these degradation equations are sufficient to consider all degradation mechanisms. Here we provide a new modeling approach to overcome these issues by digging into the concept of degradation. 
Lemma 1. The degradation phenomenon of a system is nothing but a continuous drift at least in one of the parameters of the system (Borutzky, 2014). The evolution of parameter(s) finally causes the system to fail when some parameters or a combination of parameters as a health state violate at least one predefined threshold.

Lemma 2. "Degradation phenomena can be classified into three main categories: linear, concave and convex profiles (as shown in Figure 1), and any degradation mechanism can be directly modeled by one of the bond graphs passive elements: $R$ (Resistor), $C$ (Capacitor) and I (Inductor)" (Medjaher \& Zerhouni, 2009).

As a next backup to the lemma 2, Peysson, Ouladsine, Noura, Leger, and Allemand (2008) mentioned that each degradation profile over time $\Delta$ is modeled by a two-step process, first qualitatively and then quantitatively. In their qualitative section, they characterized each degradation mechanism by the signs of its first and second derivatives $(\dot{\Delta}, \ddot{\Delta})$ by using - and + and 0 so that finally their qualitative section will be summarized just into three main profiles, linear when $\dot{\Delta} \geq 0, \ddot{\Delta}=0$, concave when $\dot{\Delta}>0, \ddot{\Delta}<0$, and convex when $\dot{\Delta}>0, \ddot{\Delta}>0$, (Figure 2). In their following work (Peysson, Ouladsine, Outbib, Leger, Myx, \& Allemand, 2009), the quantitative modeling part has been enhanced where they have characterized each degradation mechanism quantitatively by two parameters $\alpha$ and $\beta$ so that the linear degradation can be identified by $\alpha t^{\beta}$ in which $\beta=1, \alpha>0$, the concave degradation mechanisms can be identified by $\alpha t^{\beta}$ in which $0<\beta<1, \alpha>0$, and the convex degradation mechanisms can be identified by $\alpha t^{\beta}$ in which $\beta>1, \alpha>0$. In fact, the qualitative analysis identifies one of the three main profiles (linear, concave, and convex) in Figure 2, but the quantitative analysis uniquely specifies the right profile among all profiles in each three categories in Figure 2 by the values of $\alpha$ and $\beta$.

Remark 1. In real operating conditions, the equipment reliability is influenced by two sets of factors: the operating mode and the external environment (Peysson et al., 2009). In this regard, Peysson et al. (2008) identified a matrix form analysis for qualitative and quantitative investigation of degradation (Table 1 and Table 2) in which the $E_{j}$ stands for each environment condition and $O M_{i}$ stands for each operating mode. In Table 1, the first sign in each cell is related to the first derivate of degradation profile $(\dot{\Delta})$ and the second sign is related to the second derivate of the degradation profile $(\ddot{\Delta})$. So each degradation profile in Figure 2 is for one specific operating mode and one specific environment. So later, when we present our approach, it should be considered that the whole approach is valid for a given operating mode and environment, but the proposed approach can be used similarly for other operating modes and environments. In addition, Peysson et al. (2008) put only the parameter $\alpha$ of $\alpha t^{\beta}$ in Table 2 and assumed $\beta$ just can be 1 for linear, 0.5 for concave and 2 for convex and so did not put $\beta$ in Table 2, but we extended that table based on Peysson et al. (2009). Furthermore, Peysson et al. (2008), used matrix form for their own methodology, since our methodology would be different, we do not need the matrix form.

Remark 2. The constant parameters are a specific case of the linear profile with zero slope.

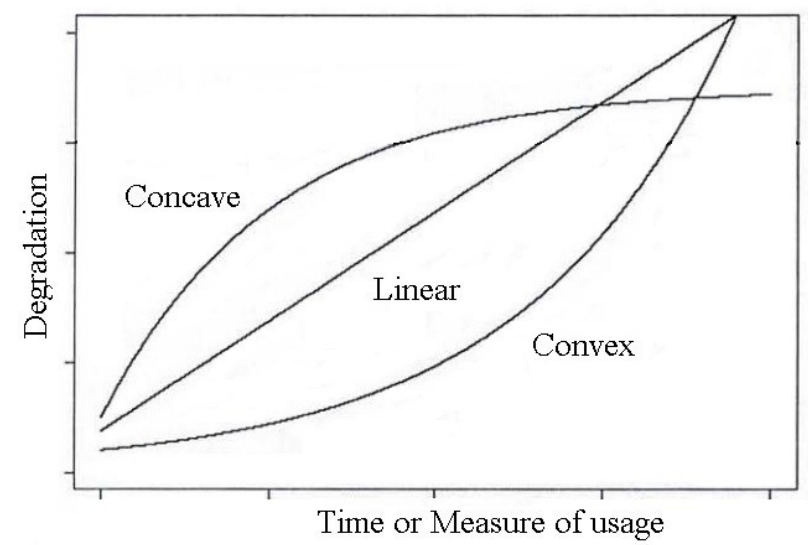

Figure 1. Possible forms for degradation models adapted from Medjaher and Zerhouni (2009)

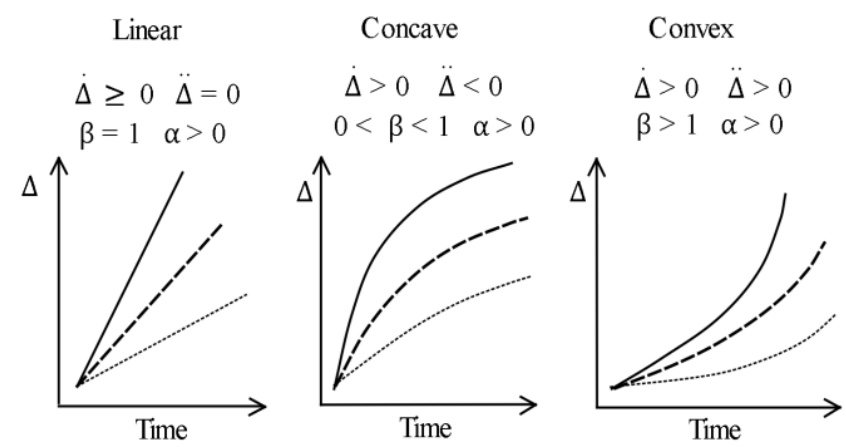

Figure 2. Qualitative and Quantitative descriptions of degradation modeling adapted from Peysson et al. (2008).

\begin{tabular}{|l|l|l|l|l|l|l|}
\hline & $E_{1}$ & $E_{2}$ & $\cdots$ & $E_{j}$ & $\cdots$ & $E_{m}$ \\
\hline$O M_{1}$ & ++ & 00 & $\cdots$ & +- & $\cdots$ & 00 \\
\hline$O M_{2}$ & +- & +0 & $\cdots$ & ++ & $\cdots$ & 00 \\
\hline$\vdots$ & $\vdots$ & $\vdots$ & $\vdots$ & $\vdots$ & $\vdots$ & $\vdots$ \\
\hline$O M_{i}$ & +0 & ++ & $\cdots$ & +0 & $\cdots$ & +- \\
\hline$\vdots$ & $\vdots$ & $\vdots$ & $\vdots$ & $\vdots$ & $\vdots$ & $\vdots$ \\
\hline$O M_{n}$ & +0 & ++ & $\cdots$ & +0 & $\cdots$ & +- \\
\hline
\end{tabular}

Table 1. The qualitative matrix adapted from Peysson et al. (2008)

\begin{tabular}{|l|l|l|l|l|l|l|}
\hline & $E_{1}$ & $E_{2}$ & $\cdots$ & $E_{j}$ & $\cdots$ & $E_{m}$ \\
\hline$O M_{1}$ & $\alpha_{1,1}$ & $\alpha_{1,2}$ & $\cdots$ & $\alpha_{1, j}$ & $\cdots$ & $\alpha_{1, m}$ \\
& $\beta_{1,1}$ & $\beta_{1,2}$ & & $\beta_{1, j}$ & & $\beta_{1, m}$ \\
\hline
\end{tabular}




\begin{tabular}{|l|l|l|l|l|l|l|}
\hline$O M_{2}$ & $\alpha_{2,1}$ & $\alpha_{2,2}$ & $\cdots$ & $\alpha_{2, j}$ & $\cdots$ & $\alpha_{2, m}$ \\
& $\beta_{2,1}$ & $\beta_{2,2}$ & & $\beta_{2, j}$ & & $\beta_{2, m}$ \\
\hline$\vdots$ & $\vdots$ & $\vdots$ & $\vdots$ & $\vdots$ & $\vdots$ & $\vdots$ \\
\hline$O M_{i}$ & $\alpha_{i, 1}$ & $\alpha_{i, 2}$ & $\cdots$ & $\alpha_{i, j}$ & $\cdots$ & $\alpha_{i, m}$ \\
& $\beta_{i, 1}$ & $\beta_{i, 2}$ & & $\beta_{i, j}$ & & $\beta_{i, m}$ \\
\hline$\vdots$ & $\vdots$ & $\vdots$ & $\vdots$ & $\vdots$ & $\vdots$ & $\vdots$ \\
\hline$O M_{n}$ & $\alpha_{n, 1}$ & $\alpha_{n, 2}$ & $\cdots$ & $\alpha_{n, j}$ & $\cdots$ & $\alpha_{n, m}$ \\
& $\beta_{n, 1}$ & $\beta_{n, 2}$ & & $\beta_{n, j}$ & & $\beta_{n, m}$ \\
\hline
\end{tabular}

Table 2. The quantitative matrix, a corrected version of Peysson et al. (2008)

Corollary 1. Based on lemmas 1 and 2, although the degradation equations in terms of inputs $(U) /$ outputs $(Y)$ / system parameters $(\Theta) /$ secondary parameters $(H)$ can have many different forms, finally they just have three main profiles with respect to time. Since degradation is nothing but drift in parameters, so each degradation equation can be represented as a system parameter being a function of time. So from a microscopic level perspective, each degradation phenomenon has a one-to-one correspondence with each system parameter (Figure 3). As we see in this figure, the number of degradation phenomena is exactly equal to the number of system parameters and each of these $k$ degradation phenomena can have one of aforementioned three profiles.

Corollary 2. The next important key point is that wear, corrosion, erosion, creep, and other well-known degradation mechanisms which are usually considered in degradation modeling, are not exactly equivalent to $e q_{1}, e q_{2}, \ldots, e q_{K}$ discussed in corollary 1 . In fact, there is not a one-to-one correspondence between $e q_{1}, e q_{2}, \ldots, e q_{K}$ and each wellknown degradation mechanism such as wear, corrosion, and etc., and actually each of wear, corrosion, and etc. can be a combination of system parameters $\theta_{1}(t), \theta_{2}(t), \ldots, \theta_{K}(t)$ and so can have more complicated graphs beyond those three main profiles. Degradation phenomenon is a more general concept than the degradation mechanism. Degradation mechanisms correspond to wear, erosion, corrosion, crack, and so forth for which, physical background have been understood, but degradation phenomenon is defined just as a drift in one system parameter. Figure 4 shows some examples of the relationship between degradation mechanisms and set of related parameters. As shown in Figure 4, the 'wear 1' can be interpreted as a drift in just one parameter while 'wear 2' and 'corrosion 1' can be a combination of several system parameters. In the latter situations, the parameters that are effective in the degradation mechanism are more than one and the profiles will not follow just one of those three profiles and can be any combinations of them like a graph with two peaks (like multimodal situations). On the other hand, some degradation phenomena like drifts in parameters 7 or 6 or 9 cannot be categorized as one of well-known degradation mechanisms like wear, corrosion, and so forth,

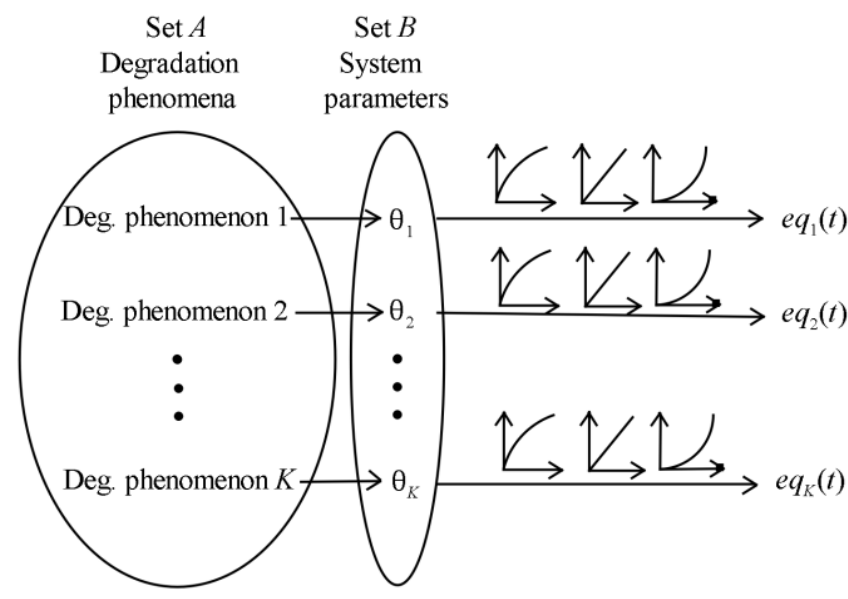

Figure 3. One-to-one correspondence (Bijection) between system degradation phenomena and system parameters.

System parameters (degradations)

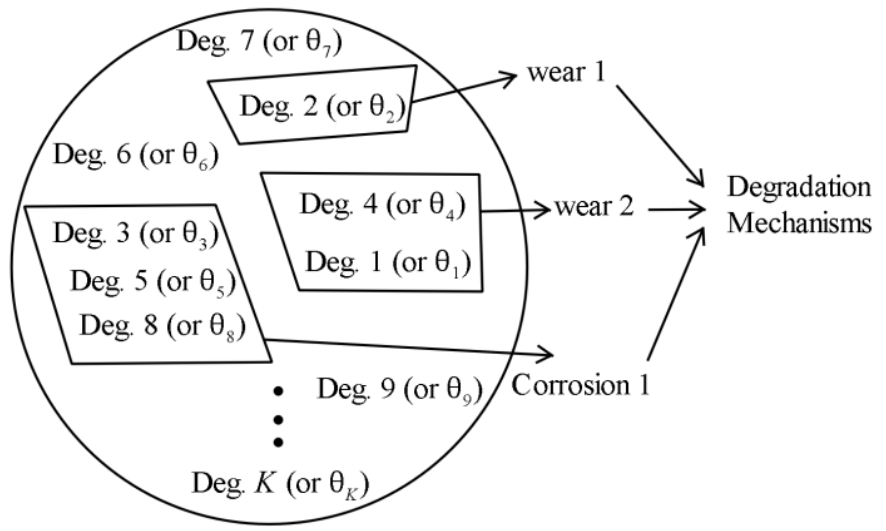

Figure 4. Illustration of system parameters and well-known degradation mechanisms.

because drifts of some system parameters do not have physical meaning or they have physical meaning, but they are unknown and unsought up to this date. These are the issues that will be solved by our approach.

Corollary 3. Most of the previous research works in parametric degradation modeling just considered the degradation mechanisms such as wear, erosion, and then used their approach for finding the parameters involved in each degradation mechanism. In these approaches, it is possible to miss some degradation phenomena like number 7 or 9, as discussed in corollary 2 in details. However, consider Figure 4, now instead of focusing on each degradation mechanism (e.g., wear 1 in which one system parameter is involved, wear 2, corrosion, etc.) to do parametric degradation modeling, we propose a new approach. We subjectively release the packages of all degradation mechanisms and remove their boundaries in Figure 4; now we have a set of all system parameters, and instead of focusing on the package of each degradation mechanism and consider the drift of each parameter. Then there are $K$ degradation phenomena microscopically, and 
each of them has just one of the three main profiles in corollary 1. In fact, in this approach, the lowest level (unit) of degradation is considered separately. By using this approach, the aforementioned issues in corollary 2 will be resolved. This is the basis of our degradation modeling. By this approach, the set of degradation equations would be more complete, and this leads to a more reliable RUL estimation.

Remark 3. It should be emphasized that based on corollaries 1, 2, and 3 our methodology can be used to model degradation phenomena for a system with a given operating mode and environment (in a similar situation and scope of previous methods), but each degradation phenomenon has only three profiles (R, I, C). These two concepts should not be mixed up. In fact, in an arbitrary system, all degradation profiles can be just linear (just one of those three profiles).

Remark 4. The decision among R, I, or C (among three profiles) could be made if run-to-failure data are available, or could be given by experts (Peysson et al. 2009). In our proposed approach we assume that data are available, so there is no need for experts.

Remark 5. In most of the previous approaches for parametric degradation modeling, based on the level of system inference, the number of degradation equations, $Q$, may change. However, in our microscopic view of degradation modeling, the number of degradation equations is always equal to the number of system parameters, $K$.

\section{PROVIDING A GENERIC FRAMEWORK BY COMBINING THE GOVERNING EQUATIONS AND DEGRADATION EQUATIONS}

In our approach, the form of primary (governing) equations is similar to Eq. (1) however, the form of secondary (degradation) equations is different from Eq. (3) and is like Eq. (9). In fact, in parametric degradation modeling, finding the form of degradation equations has been always a challenge, because degradation can be modeled as a function of inputs $(U)$ / outputs $(Y)$ / system parameters $(\Theta)$ / secondary parameters $(H)$, but we resolved this challenge by a new approach for degradation modeling and we found that the degradation equations based on a microscopic approach can be written like Eq. (9), arising from Figure 3. Now we are going to describe the structured way to find them in the current section.

$$
\left\{\begin{array}{c}
e q_{P+1}=e q_{P+1}\left(\theta_{1}(t)\right) \\
e q_{P+2}=e q_{P+2}\left(\theta_{2}(t)\right) \\
\cdot \\
\cdot \\
e q_{P+K}=e q_{P+K}\left(\theta_{K}(t)\right),
\end{array}\right.
$$

In Eq. (9), coefficients need to be estimated in the process of parameter model fitting. Also, some parameters will be found to be constant like the mass in the mass-springdamper system.

Up to here we found the final forms of degradation equations over time, now consider the following logical sequence:

1. Model order and structure are determined.

2. Model identification techniques (section 3.2) are used to find the constant parameters, but:

3. In PHM-related works, run to failure cases are in one's interest, so parameters are variable rather than constant.

4. So, in order to use model identification techniques, the run-to-failure time is divided into certain number of intervals of time such that the parameters can be considered constant in each interval, then the model identification techniques can be used to find the constant parameters for each interval, but since the model structure and the model order might be different in each interval, it is better we make the time intervals before step 1 .

5. After implementation of the preceding steps for all intervals and all parameters, the value of each parameter for the run-to-failure period can be attained.

6. Fitting parameters to one of $I, C$, or $R$.

7. After implementing the preceding step for all parameters, degradation equations are derived that govern the parameters evolution over time.

8. Now all governing equations of the system are derived.

9. Finally, RUL or EOL estimation can be proceeded.

Remark 6. In the physics-based approach, before proceeding the RUL or EOL estimation, we need to substitute the values for some constant parameters like masses, material properties and etc., while in the proposed generic approach, such parameter substitution is not required, because they will be found by having data and using system identification techniques organized in section 3.

\section{Discussion}

We have revisited the degradation phenomenon and concluded the corollary 1,2, and 3 and combined them in a new way with some system identification techniques to finally provide a generic degradation modeling (which is coupled with governing equations). 
This approach is different from the physics-based approach. Also, as found in the literature, most of the parametric datadriven approaches do not have a generic approach to (1) derive governing equations, (2) derive degradation equations, and (3) finally combine them in a way so that the generality is conserved. The proposed approach is comprehensive because it can provide both system and degradation equations (primary and secondary equations) in a generic way and covers nonlinear systems as well. In other approaches, there is no guaranty that all degradation equations are formulated, and there is always the possibility that a specific degradation phenomenon of a system is unknown or partially known and consequently it will be ignored. However, our microscopic approach in degradation modeling based on corollaries 1, 2, and 3 can be more reliable.

To summarize the modeling framework and its steps, the flowchart of the proposed approach is illustrated in Figure 5.

Finally, to illustrate the capability of this method in practice, the comparison between previous studies and the proposed approach in parametric degradation modeling are discussed. Consider a centrifugal pump and bearing system (Figure 6) which has been studied by the traditional parametric degradation modeling researchers (Daigle et al., 2012). In order to find the remaining useful life of this system, the researchers need to find the degradation models. Conventional approaches utilize a physical understanding of the system to identify some critical components that are more prone to degrade over time. One of the main degradation mechanisms of the centrifugal pump is mechanical wear of bearing due to sliding and rolling friction. Hence, they chose two appropriate degradation models from the previous studies such as Eq. (10) (Daigle et al., 2012):

$$
\left\{\begin{array}{l}
\dot{r_{t}}(t)=w_{t} r_{t} \omega^{2} \\
\dot{r_{r}}(t)=w_{r} r_{r} \omega^{2},
\end{array}\right.
$$

where $\omega$ is the rotational velocity of the pump, $r_{t}$ is the friction coefficient for the thrust bearings, $r_{r}$ is the friction coefficient for the radial bearings, $w_{t}$ and $w_{r}$ are the wear coefficients (Daigle et al., 2012).

This approach for parametric degradation modeling works relatively well until we face two main situations:

1. For complex engineering systems which are fabricated by the same conventional manufacturing processes but composed lots of components and a variety of degradation mechanisms, finding the appropriate degradation models is not as straightforward as the aforementioned simple case,

2. The structures which are fabricated by the new manufacturing processes such as 4D printing which do not have any conventional mechanical and electromechanical parts such as bearings and so forth but will undergo the degradation such as Figure 7.

The difficulty of the current parametric degradation modeling for the first situation which is an extension of the aforementioned simple case would be evident by a priori knowledge. However, for the second situation consider the Figure 7 showing a simple case of 4D printed structure. Similar to the other 4D printed structures, it does not have any conventional parts like bearings, electromechanical parts, and so forth. It is just a multi-material, single-piece, one-time printed structure but will undergo the degradation. The current parametric degradation modeling would not be totally suitable for this situation as well. However, the proposed approach in this paper by introducing the degradation phenomenon as a more general concept than degradation mechanism, leading to a general methodology, would be more reliable for these two situations. 


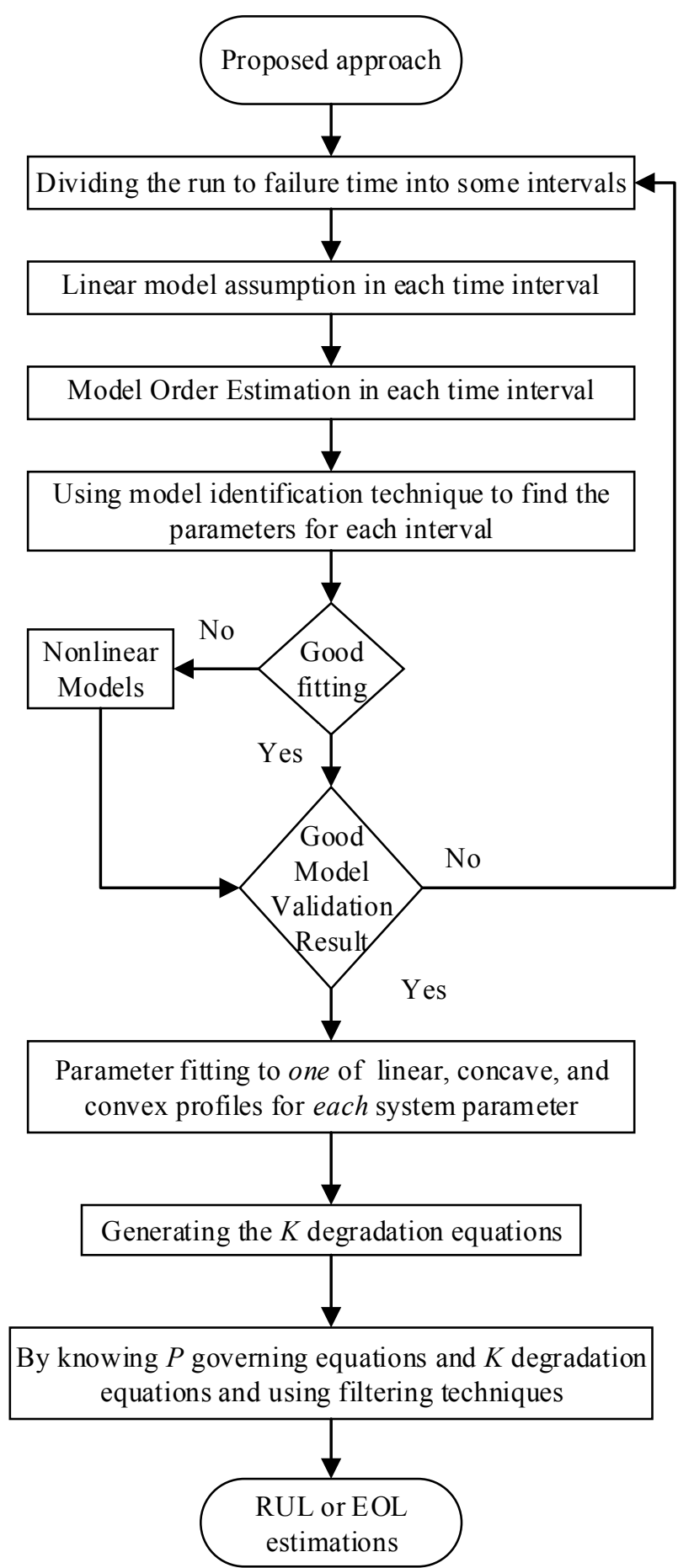

Figure 5. Flowchart of proposed approach

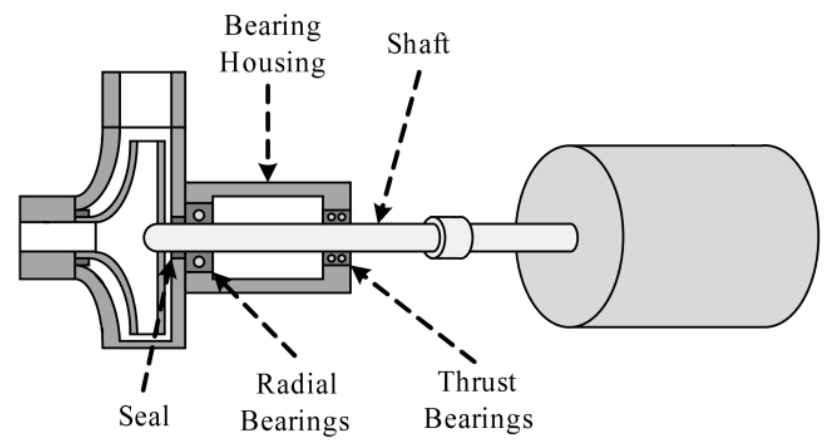

Figure 6. Centrifugal pump and its bearings adapted from Daigle et al. (2012).

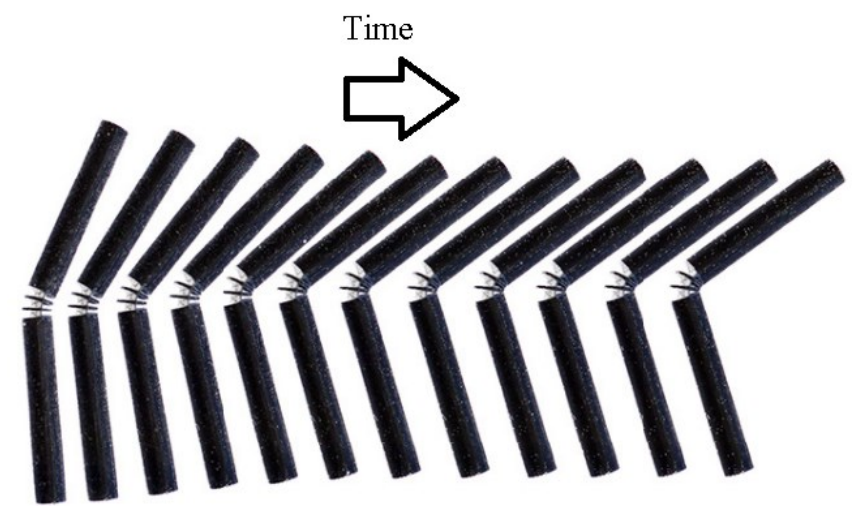

Figure 7. 4D printed structure performing the shape-shifting under water, over time, adapted from Tibbits et al. (2014).

\section{CONCLUSION}

We addressed and organized a new approach in degradation modeling. This approach can be more beneficial in the cases that the physical knowledge is not sufficient such as two main situations including: (1) Complex engineering systems which are fabricated by the conventional manufacturing processes but consists of numerous components and various degradation mechanisms, (2) the structures which are created by the new manufacturing processes such as 4D printing which do not have any conventional mechanical and electromechanical parts but will undergo the degradation. The major aspects of this study are (1) quantifying the concepts of both system governing equations and degradation equations in prognostics in a general form, (2) providing a generic approach to derive the governing equations by using some techniques from system identification, (3) analyzing the concepts of degradation so that it provided a microscopic approach for generic degradation modeling, and (4) incorporating the governing equations and degradation equations in a way that the generality of the approach for finding governing equations and degradation equations is conserved. The main contributions of this research lie in degradation modeling and combining it with governing equations by a generic perspective. The proposed approach due to its nature can be 
engaged in system-level degradation modeling with partial physical knowledge for resolving prognostic problems in new and complex systems.

\section{REFERENCES}

Aizpurua, J. I., \& Catterson, V. M. (2015). Towards a Methodology for Design of Prognostic Systems. In Annual Conference of the Prognostics and Health Management Society, Coronado, California, USA. Available:

https://www.phmsociety.org/sites/phmsociety.org/files/ phm_submission/2015/phmc_15_022.pdf

Borutzky, W. (2014). Failure Prognosis for Hybrid Systems Based on ARR Residuals. Bond Graph Model-based Fault Diagnosis of Hybrid Systems, 221-233. doi:10.1007/978-3-319-11860-4 9.

Daigle, M. J., \& Goebel, K. (2011). A model-based prognostics approach applied to pneumatic valves. International Journal of Prognostics and Health Management Volume 2 (color), 84. Available: http://ntrs.nasa.gov/archive/nasa/casi.ntrs.nasa.gov/201 20002692.pdf

Daigle, M., Saha, B., \& Goebel, K. (2012). A comparison of filter-based approaches for model-based prognostics. In Aerospace Conference, 2012 IEEE, 1-10. doi:10.1109/aero.2012.6187363.

Garnier, H., Mensler, M., \& Richard, A. (2003). Continuous-time model identification from sampled data: Implementation issues and performance evaluation. International Journal of Control, 76(13), 1337-1357. doi:10.1080/0020717031000149636.

Liao, L., \& Kottig, F. (2014). Review of Hybrid Prognostics Approaches for Remaining Useful Life Prediction of Engineered Systems, and an Application to Battery Life Prediction. IEEE Transactions on Reliability IEEE Trans. Rel., 63(1), 191-207. doi:10.1109/tr.2014.2299152.

Ljung, L. (1999). System Identification: theory for the user. Upper Saddle River, NJ: Prentice Hall PTR.

Ljung, L. (2009). Experiments with Identification of Continuous Time Models. IFAC Proceedings Volumes, 42(10), 1175-1180. doi:10.3182/20090706-3-fr2004.00195.

Ljung, L. (2013). System Identification Toolbox: User's Guide. The Mathworks Inc., Natick, MA. [Online]. Available:www.mathworks.com/help/pdf_doc/ident/ide nt.pdf.

Medjaher, K., \& Zerhouni, N. (2009). Residual-based failure prognostic in dynamic systems. IFAC Proceedings Volumes, 42(8), 716-721. <hal-00402959> doi:10.3182/20090630-4-es-2003.00119.

Peysson, F., Ouladsine, M., Noura, H., Leger, J., \& Allemand, C. (2008). New Approach to Prognostic System Failures. IFAC Proceedings Volumes, 41(2), 12861-12866. doi:10.3182/20080706-5-kr-1001.02175.
Peysson, F., Ouladsine, M., Outbib, R., Leger, J., Myx, O., \& Allemand, C. (2009). A Generic Prognostic Methodology Using Damage Trajectory Models. IEEE Transactions on Reliability IEEE Trans. Rel., 58(2), 277-285. doi:10.1109/tr.2009.2020123.

Raviv, D., Zhao, W., McKnelly, C., Papadopoulou, A., Kadambi, A., Shi, B., Hirsch, S., Dikovsky, D., Zyracki, M., Olguin, C., Raskar, R., \& Tibbits, S. (2014). Active Printed Materials for Complex SelfEvolving Deformations. Sci. Rep. Scientific Reports, 4, 7422. doi:10.1038/srep07422.

Tibbits, S. (2014). 4D Printing: Multi-Material Shape Change. Architectural Design Archit Design, 84(1), 116-121. doi:10.1002/ad.1710

Tibbits, S., McKnelly, C., Olguin, C., Dikovsky, D., Hirsch, S. (2014). 4D Printing and Universal Transformation. Proceedings of the Association for Computer Aided Design in Architecture 2014 Los Angeles, CA, 539-548. Available: http://papers.cumincad.org/data/works/att/acadia14_539 .content.pdf 